Research Article

\title{
Mean Arterial Pressure Is Related to Incident Nonalcoholic Fatty Liver Disease among the Nonobese Female with Normal Low-Density Lipoprotein Cholesterol Levels: A Large Cohort Study in China
}

\author{
Shangbo Xu, ${ }^{1}$ Lan Chen, ${ }^{1}$ Danhua Hong, ${ }^{1}$ Lihua Yang, ${ }^{2}$ Xiaozhi Li, ${ }^{3}$ and Xin Wang ${ }^{1}$ \\ ${ }^{1}$ Department of Cardiology, First Affiliated Hospital of Shantou University Medical College, No. 57 of Changping Road, Shantou, \\ Guangdong 515041, China \\ ${ }^{2}$ Department of Nephrology, First Affiliated Hospital of Shantou University Medical College, No. 57 of Changping Road, Shantou, \\ Guangdong 515041, China \\ ${ }^{3}$ Department of Oncology, First Affiliated Hospital of Shantou University Medical College, No. 57 of Changping Road, Shantou, \\ Guangdong 515041, China
}

Correspondence should be addressed to Xin Wang; xusb2012@163.com

Received 3 October 2019; Accepted 6 January 2020; Published 17 January 2020

Academic Editor: Mario Pirisi

Copyright (C) 2020 Shangbo Xu et al. This is an open access article distributed under the Creative Commons Attribution License, which permits unrestricted use, distribution, and reproduction in any medium, provided the original work is properly cited.

\begin{abstract}
Aim. We aimed to demonstrate the independent effect of mean arterial pressure (MAP) on incident nonalcoholic fatty liver disease (NAFLD) among the nonobese Chinese with normal low-density lipoprotein cholesterol (LDL-C) levels. Methods. 16,153 nonobese participants without NAFLD at baseline were enrolled and then assigned to four groups by quartiles of MAP (Q1-Q4). A subgroup analysis by gender was also conducted. Participants were diagnosed with NAFLD by ultrasonography. Results. During a mean follow-up of 2.80 years, the cumulative incidence of NAFLD was 14.37 and the incidence rate was 513.17 per 10,000 personyears. The cumulative incidence of NAFLD for the whole population or gender groups gradually increased with the quartiles of MAP (all $P<0.001$ ). In the Q4 of MAP, the cumulative incidence of NAFLD for the whole population, male, and female reached up to 6.22 (5.75-6.70), 6.70 (6.21-7.19), and 5.69 (5.24-6.14), respectively. After adjustment for potential confounders, as compared with Q1, the hazard ratio for NAFLD was 1.328 (1.072-1.647), 1.625 (1.276-2.069), and 1.697 (1.231-2.340) for Q2, Q3, and Q4, respectively. In subgroup analysis, the respective hazard ratio for NAFLD in Q2, Q3, and Q4 of MAP was 1.760 (1.276-2.429), 2.080 (1.433-3.019), and 2.377 (1.452-3.890), compared with female in the Q1 of MAP. But MAP was not associated with incident NAFLD in male. Besides, MAP had a larger area under the receiver-operating characteristic curves than SBP or DBP, with optimal cutoff point of $88 \mathrm{mmHg}$ in male and $89 \mathrm{mmHg}$ in female. Conclusions. MAP is an independent predictor for incident NAFLD among nonobese female with normal LDL levels.
\end{abstract}

\section{Introduction}

Nonalcoholic fatty liver disease (NAFLD) is recognized as a common spectrum liver disease, encompassing nonalcoholic fatty liver (simple steatosis) and nonalcoholic steatohepatitis (NASH), which may progress to cirrhosis even hepatocellular carcinoma at last [1]. Owing to the epidemic of obesity, the prevalence of NAFLD has been increasing dramatically and was reported approximately $25-30 \%$ of the general population worldwide $[1,2]$, including Asia-Pacific region which was thought to be a nonepidemic area in the past [3]. Furthermore, it has been demonstrated that NAFLD is a significant risk factor for developing hypertension, type 2 diabetes mellitus, and cardiovascular disease [4-6]. Therefore, it is quite interesting to identify individuals at a high risk of developing NAFLD. 
Blood pressure (BP), including systolic blood pressure (SBP) and diastolic blood pressure (DBP), is an important risk factor for developing NAFLD [7-10], even in individuals without hypertension [10]. Mean arterial pressure (MAP), a steady component of BP, has wildly been used in clinical practice. However, no study is available with respect to the association between MAP and incident NAFLD. For another, most previous studies paid their attention to progression of NAFLD among the obese population. And a previous study enrolled 183,903 nonobese Chinese with normal lowdensity lipoprotein cholesterol (LDL-C) levels, of which 25,503 individuals were diagnosed with NAFLD by ultrasonography [11].

Our study therefore is aimed at demonstrating the association between MAP and incident NAFLD among a nonobese population with normal LDL-C levels in China.

\section{Materials and Methods}

2.1. Study Design and Participants. The raw data that we tried to analyze was downloaded freely from an online database named "DATADRYAD” (http://www.Datadryad.org), which supports the reuse of data of published studies. In the light of terms of service, we cited the data package upload by Sun et al. in our present study $[11,12]$. We performed a secondary analysis with no need for another ethic approval because the original study protocol was vetted and approved by the ethics committee of Wenzhou People's Hospital. The participants in the original study were individuals who attended a health examination from January 2010 to December 2014. Participants would undergo annual evaluation throughout the follow-up period. Individuals were excluded if they met the following criteria: (1) excess consumption of alcohol (more than $140 \mathrm{~g} /$ week for male and $70 \mathrm{~g} /$ week for female); (2) any known causes of chronic hepatic disease, such as viral hepatitis or autoimmune hepatitis; (3) a body mass index (BMI) equal to or more than $25 \mathrm{~kg} / \mathrm{m}^{2}$; (4) a high level of LDL-C (>3.12 mmol/L); (5) those taking antihypertensive, antidiabetic, or lipidlowering agents; and (6) lost to follow-up or with missing data. At the same time, we eliminated 20 participants without available SBP and/or DBP. Finally, a total of 16,153 participants without NAFLD at the baseline were included in our research. The process of the selection was specifically exposited in the previous report [11].

2.2. Data Collection. The following variables included in the data package were extracted: gender, age, body mass index (BMI), SBP, DBP, alkaline phosphatase (ALP), alanine aminotransferase (ALT), aspartate aminotransferase (AST), albumin (ALB), globulin (GLB), total bilirubin (TB), blood urea nitrogen (BUN), creatinine (Cr), uric acid (UA), fasting plasma glucose (FPG), total cholesterol (TC), triglyceride (TG), high-density lipoprotein cholesterol (HDL-C), LDLC, duration of follow-up, and outcome of follow-up. The trained medical staff used a standardized procedure to obtain participant's medical history and health habit. BP was measured with an automated sphygmomanometer with the subjects in a sitting position and a quiet environment. Fasting venous blood sample collection (required an overnight fast) would be measured with an automated analyzer (Abbott AxSYM). More specific details were presented in the previous reports $[8,11]$.

2.3. Definitions and Outcomes. BMI was calculated as the weight $(\mathrm{kg})$ to the height $\left(\mathrm{m}^{2}\right)$, and MAP was calculated as $[\mathrm{SBP}+(2 \times \mathrm{DBP})] / 3$. Participants were diagnosed with NAFLD by ultrasonography according to the recommendations of the Chinese Liver Disease Association [13]. Generally speaking, NAFLD was diagnosed as diffuse enhancement of the close field echo in the hepatic region (greater than in the region of the kidney and spleen) and gradually attenuated beam in the far field echo, if in combination with one of the following items: intrahepatic lacuna structure is unclearly displayed; a round and blunt border in mild-to-moderate hepatomegaly; a decrease of the blood flow signal, while the distribution of blood flow is still normal; and the display of envelop of the right liver lobe and diaphragm is unclear or nonintact.

2.4. Statistical Analysis. All participants were classified into four groups based on the quartiles of baseline MAP: Q1 (58 to $<80 \mathrm{mmHg}$ ), Q2 (80 to $<88 \mathrm{mmHg}$ ), Q3 (88 to $<96 \mathrm{mmHg}$ ), and Q4 (96 to $\leq 142 \mathrm{mmHg}$ ). Continuous variables were expressed as mean \pm standard deviation (SD), while categorical variables were expressed as number (percentage). The one-way ANOVA and chi-squared test were used to determine any statistical differences of baseline characteristics for the continuous and categorical variables, respectively. Meanwhile, differences of baseline data between male and female were performed. Then, we calculated the incidence rate (per 10,000 person-years) and cumulative incidence with 95\% confidence interval (CI) of NAFLD. Cumulative hazard curves were plotted using the KaplanMeier method, and the log-rank test was used to compare the cumulative incidence of NAFLD stratified by MAP. We performed the cox's proportional hazard regression analyses to evaluate the association of MAP with the incident NAFLD. Hazard ratio (HR) with a 95\% CI was reported. We also conducted a subgroup analysis stratified by gender. Receiveroperating characteristic (ROC) curve was used to compare the abilities of SBP, DBP, and MAP in predicting the incident NAFLD. The optimal cutoff of MAP was calculated according to the highest Youden index (sensitivity+specificity-1). A $P<0.05$ (two tailed) was considered statistically significant. All analyses involved the use of SPSS V.25.0 software (SPSS Inc., Chicago, Illinois, USA).

\section{Results}

3.1. Baseline Characteristics of Participants. We included a total of 16,153 participants $(8,472$ men, $52.4 \%)$ who were free of NAFLD at baseline. The mean age of this population was 43.23 years, and the MAP ranged from 58 to $142 \mathrm{mmHg}$. The baseline characteristics of participants by MAP quartiles are shown in Table 1. Age, the number of males, BMI, SBP, DBP, ALP, ALT, AST, ALB, GLB, TB, BUN, Cr, UA, FPG, TC, TG, and LDL-C all tended to be higher in the higher 
TABLE 1: Baseline characteristics of 16,153 participants by mean arterial pressure quartiles.

\begin{tabular}{|c|c|c|c|c|c|}
\hline Characteristics & $58 \leq \mathrm{Q} 1<80 \mathrm{mmHg}$ & $80 \leq \mathrm{Q} 2<88 \mathrm{mmHg}$ & $88 \leq \mathrm{Q} 3<96 \mathrm{mmHg}$ & $96 \leq \mathrm{Q} 4 \leq 142 \mathrm{mmHg}$ & $P$ value \\
\hline Number (\%) & $3,806(23.6)$ & $4,414(27.3)$ & $3,744(23.2)$ & $4,189(25.9)$ & \\
\hline Age (years) & $41.66 \pm 14.32$ & $42.32 \pm 14.64$ & $43.29 \pm 15.04$ & $45.55 \pm 15.50$ & $<0.001$ \\
\hline Male, $N(\%)$ & $1,817(47.7)$ & $2,247(50.9)$ & $2,049(54.7)$ & $2,359(56.3)$ & $<0.001$ \\
\hline BMI $\left(\mathrm{kg} / \mathrm{m}^{2}\right)$ & $20.45 \pm 1.94$ & $21.15 \pm 1.98$ & $21.67 \pm 1.95$ & $22.20 \pm 1.90$ & $<0.001$ \\
\hline SBP (mmHg) & $102.53 \pm 6.79$ & $114.29 \pm 6.41$ & $124.08 \pm 7.69$ & $141.05 \pm 13.11$ & $<0.001$ \\
\hline $\mathrm{DBP}(\mathrm{mmHg})$ & $60.88 \pm 3.91$ & $68.67 \pm 3.51$ & $75.43 \pm 4.11$ & $85.68 \pm 7.21$ & $<0.001$ \\
\hline ALP (U/L) & $65.22 \pm 21.44$ & $70.20 \pm 21.60$ & $73.79 \pm 25.16$ & $77.92 \pm 22.65$ & $<0.001$ \\
\hline $\operatorname{ALT}(\mathrm{U} / \mathrm{L})$ & $16.71 \pm 12.14$ & $19.59 \pm 18.48$ & $21.05 \pm 15.26$ & $21.91 \pm 17.51$ & $<0.001$ \\
\hline AST (U/L) & $20.79 \pm 7.73$ & $22.34 \pm 9.80$ & $23.48 \pm 8.11$ & $24.82 \pm 10.88$ & $<0.001$ \\
\hline $\operatorname{ALB}(g / L)$ & $44.01 \pm 2.49$ & $44.46 \pm 2.71$ & $44.65 \pm 2.75$ & $44.46 \pm 2.83$ & $<0.001$ \\
\hline $\mathrm{GLB}(\mathrm{g} / \mathrm{L})$ & $29.32 \pm 3.55$ & $29.33 \pm 3.92$ & $29.36 \pm 3.78$ & $29.95 \pm 4.11$ & $<0.001$ \\
\hline $\mathrm{TB}(\mu \mathrm{mol} / \mathrm{L})$ & $11.50 \pm 4.50$ & $12.07 \pm 5.24$ & $12.28 \pm 4.89$ & $12.53 \pm 5.00$ & $<0.001$ \\
\hline $\mathrm{BUN}(\mathrm{mmol} / \mathrm{L})$ & $4.25 \pm 1.13$ & $4.46 \pm 1.24$ & $4.64 \pm 1.39$ & $4.90 \pm 1.58$ & $<0.001$ \\
\hline $\mathrm{Cr}(\mathrm{mmol} / \mathrm{L})$ & $70.62 \pm 15.49$ & $76.21 \pm 17.82$ & $81.14 \pm 24.78$ & $85.66 \pm 36.40$ & $<0.001$ \\
\hline $\mathrm{UA}(\mu \mathrm{mol} / \mathrm{L})$ & $244.36 \pm 72.24$ & $270.37 \pm 81.13$ & $291.36 \pm 86.11$ & $311.70 \pm 88.33$ & $<0.001$ \\
\hline FPG (mmol/L) & $4.91 \pm 0.52$ & $5.03 \pm 0.63$ & $5.18 \pm 0.80$ & $5.44 \pm 0.98$ & $<0.001$ \\
\hline $\mathrm{TC}(\mathrm{mmol} / \mathrm{L})$ & $4.47 \pm 0.71$ & $4.57 \pm 0.72$ & $4.67 \pm 0.75$ & $4.78 \pm 0.76$ & $<0.001$ \\
\hline $\mathrm{TG}(\mathrm{mmol} / \mathrm{L})$ & $1.03 \pm 0.54$ & $1.21 \pm 0.78$ & $1.38 \pm 0.93$ & $1.57 \pm 1.18$ & $<0.001$ \\
\hline $\mathrm{HDL}-\mathrm{C}(\mathrm{mmol} / \mathrm{L})$ & $1.51 \pm 0.35$ & $1.48 \pm 0.36$ & $1.44 \pm 0.37$ & $1.42 \pm 0.37$ & $<0.001$ \\
\hline LDL-C (mmol/L) & $2.16 \pm 0.46$ & $2.24 \pm 0.46$ & $2.30 \pm 0.46$ & $2.35 \pm 0.46$ & $<0.001$ \\
\hline Duration of follow up (years) & $2.89 \pm 1.11$ & $2.76 \pm 1.13$ & $2.82 \pm 1.14$ & $2.75 \pm 1.19$ & $<0.001$ \\
\hline NAFLD (\%) & $191(5.0)$ & $473(10.7)$ & $652(17.4)$ & $1,005(24.0)$ & $<0.001$ \\
\hline
\end{tabular}

Note: continuous variables were described by mean \pm standard deviation, and categorical variables were presented by number (percentage). Abbreviations: $\mathrm{BMI}=$ body mass index; $\mathrm{SBP}=$ systolic blood pressure; $\mathrm{DBP}=$ diastolic blood pressure; $\mathrm{ALP}=$ alkaline phosphatase; $\mathrm{ALT}=$ alanine aminotransferase; $\mathrm{AST}=$ aspartate aminotransferase; $\mathrm{ALB}=$ albumin $; \mathrm{GLB}=$ globulin $; \mathrm{TB}=$ total bilirubin; $\mathrm{BUN}=$ blood urea nitrogen; $\mathrm{Cr}=\mathrm{creatinine} ; \mathrm{UA}=\mathrm{uric}$ acid; $\mathrm{FPG}=$ fasting plasma glucose; $\mathrm{TC}=$ total cholesterol; $\mathrm{TG}=$ triglyceride; HDL-C $=$ high density lipoprotein cholesterol; LDL-C $=$ low-density lipoprotein cholesterol; NAFLD = nonalcoholic fatty liver disease.

MAP compared with the lower MAP $(P<0.001)$, but HDL-C changed in the opposite trend simultaneously $(P<0.001)$. Then, an analysis stratified by gender was also conducted and is summarized in Table 2. Compared with female, male was older, with higher BMI, SBP, DBP, ALP, ALT, AST, ALB, TB, BUN, Cr, UA, FPG, TG and the incidence rate of NAFLD, and lower HDL-C. However, no significant differences were observed in MAP, GLB, TC, and LDL-C between female and male.

3.2. Incidence Rate of NAFLD. During a mean follow-up of 2.80 years, 2,321 participants developed new-onset NAFLD (Table 3). Overall, the cumulative incidence of NAFLD was $14.37(13.68-15.06)$ and the incidence rate was 513.17 per 10,000 person-years. The cumulative incidence of NAFLD for the whole population or gender groups gradually increased with the quartiles of MAP (all $P<0.001$ ). In the Q4 of MAP, the cumulative incidence of NAFLD for the whole population, male, and female reached up to 6.22 (5.75-6.70), 6.70 (6.21-7.19), and 5.69 (5.24-6.14), respectively. Besides, both incidence rate and cumulative incidence were higher in male than in female. Figure 1 shows the cumulative incidence of NAFLD stratified by MAP.
3.3. The Association of MAP with the Incident NAFLD. To evaluate the association of MAP with the incident NAFLD, Cox's proportional hazard regression analyses were applied. Table 4 summarizes the specific results of the univariate cox analysis and multivariate cox analysis. There was a positive association of MAP with NAFLD in the univariate cox analysis $(P$ for trend $<0.001)$. As compared with $Q 1$, the HR for NAFLD was 2.273 (1.921-2.689), 3.613 (3.0754.245), and 5.083 (4.354-5.934) for Q2, Q3, and Q4, respectively. Then, a multivariate cox analysis was performed by including variables with a $P$ value less than 0.2 in the univariate cox analysis, after adjustment for age, gender, BMI, SBP, DBP, ALP, ALT, AST, GLB, BUN, Cr, UA, FPG, TG, TC, TG, HDL-C, and LDL-C and the positive association of MAP with incident NAFLD remained significant $(P$ for trend $<$ 0.001). Similarly, compared with Q1, the multivariate HR for incident NAFLD was diminished but remained significant in Q2, Q3, and Q4, and the specific values for Q2, Q3, and Q4 were as follows: 1.328 (1.072-1.647), 1.625 (1.2762.069), and 1.697 (1.231-2.340).

3.4. Subgroup Analysis by Gender. In order to verify the robustness of the combined effect of gender and MAP on 
TABLE 2: Baseline characteristics of 16,153 participants stratified by gender.

\begin{tabular}{|c|c|c|c|}
\hline Characteristics & Female & Male & $P$ value \\
\hline Number (\%) & $7,681(47.6)$ & $8,472(52.4)$ & \\
\hline Age (years) & $40.37 \pm 12.72$ & $45.82 \pm 16.31$ & $<0.001$ \\
\hline BMI $\left(\mathrm{kg} / \mathrm{m}^{2}\right)$ & $21.26 \pm 2.02$ & $21.50 \pm 2.07$ & $<0.001$ \\
\hline SBP (mmHg) & $119.62 \pm 16.59$ & $121.74 \pm 16.77$ & $<0.001$ \\
\hline DBP (mmHg) & $72.14 \pm 10.18$ & $73.42 \pm 10.47$ & $<0.001$ \\
\hline MAP (mmHg) & $87.96 \pm 11.46$ & $89.53 \pm 11.67$ & 0.139 \\
\hline ALP (U/L) & $71.06 \pm 22.49$ & $73.41 \pm 23.77$ & $<0.001$ \\
\hline $\operatorname{ALT}(\mathrm{U} / \mathrm{L})$ & $19.68 \pm 18.19$ & $20.37 \pm 14.86$ & 0.022 \\
\hline AST (U/L) & $22.83 \pm 9.99$ & $23.21 \pm 9.08$ & 0.031 \\
\hline $\operatorname{ALB}(g / L)$ & $44.28 \pm 2.63$ & $44.51 \pm 2.77$ & $<0.001$ \\
\hline GLB (g/L) & $29.56 \pm 3.86$ & $29.44 \pm 3.87$ & 0.068 \\
\hline $\mathrm{TB}(\mu \mathrm{mol} / \mathrm{L})$ & $11.87 \pm 4.72$ & $12.34 \pm 5.13$ & $<0.001$ \\
\hline $\mathrm{BUN}(\mathrm{mmol} / \mathrm{L})$ & $4.52 \pm 1.36$ & $4.61 \pm 1.37$ & $<0.001$ \\
\hline $\mathrm{Cr}(\mathrm{mmol} / \mathrm{L})$ & $76.55 \pm 25.45$ & $80.24 \pm 25.79$ & $<0.001$ \\
\hline $\mathrm{UA}(\mu \mathrm{mol} / \mathrm{L})$ & $271.72 \pm 84.41$ & $287.17 \pm 86.64$ & $<0.001$ \\
\hline $\mathrm{FPG}(\mathrm{mmol} / \mathrm{L})$ & $5.12 \pm 0.80$ & $5.16 \pm 0.76$ & 0.002 \\
\hline $\mathrm{TC}(\mathrm{mmol} / \mathrm{L})$ & $4.62 \pm 0.74$ & $4.62 \pm 0.75$ & 0.971 \\
\hline $\mathrm{TG}(\mathrm{mmol} / \mathrm{L})$ & $1.25 \pm 0.79$ & $1.35 \pm 1.01$ & $<0.001$ \\
\hline HDL-C (mmol/L) & $1.48 \pm 0.35$ & $1.45 \pm 0.36$ & $<0.001$ \\
\hline LDL-C (mmol/L) & $2.26 \pm 0.46$ & $2.27 \pm 0.47$ & 0.125 \\
\hline Duration of follow-up (years) & $2.85 \pm 1.12$ & $2.76 \pm 1.16$ & $<0.001$ \\
\hline NAFLD (\%) & $1,027(13.4)$ & $1,294(15.3)$ & 0.001 \\
\hline
\end{tabular}

Note: continuous variables were described by mean \pm standard deviation, and categorical variables were presented by number (percentage). Abbreviations: $\mathrm{BMI}=$ body mass index; $\mathrm{SBP}=$ systolic blood pressure; $\mathrm{DBP}=$ diastolic blood pressure; $\mathrm{MAP}=$ mean arterial pressure; $\mathrm{ALP}=$ alkaline phosphatase; $\mathrm{ALT}=$ alanine aminotransferase; $\mathrm{AST}=$ aspartate aminotransferase; $\mathrm{ALB}=$ albumin; $\mathrm{GLB}=$ globulin; $\mathrm{TB}=$ total bilirubin; $\mathrm{BUN}=$ blood urea nitrogen; $\mathrm{Cr}=$ creatinine; $\mathrm{UA}=$ uric acid; $\mathrm{FPG}=$ fasting plasma glucose; $\mathrm{TC}=$ total cholesterol; $\mathrm{TG}=$ triglyceride; HDL-C=high density lipoprotein cholesterol; LDL-C = low-density lipoprotein cholesterol; NAFLD = nonalcoholic fatty liver disease.

incident NAFLD, the HR of incident NAFLD stratified into 2 groups of gender (male and female) is presented in Table 5. For female, after further adjustment for age, BMI, SBP, DBP, ALP, ALT, AST, GLB, BUN, Cr, UA, FPG, TG, TC, TG, HDL-C, and LDL-C, the risk of developing NAFLD gradually increased with quartiles of the MAP ( $P$ for trend $=0.002)$. The respective HR for NAFLD in Q2, Q3, and Q4 of MAP were 1.760 (1.276-2.429), 2.080 (1.433-3.019), and 2.377 (1.452-3.890), compared with female in the Q1 of MAP. In contrast, MAP was not associated with the risk of developing NAFLD in male $(P>0.05)$.

3.5. ROC Analysis of MAP and Risks for NAFLD. As shown in Figures 2 and 3, SBP, DBP, and MAP were significant predictors for future risk of NAFLD in the group of male or female (all $P<0.05$ ). The area under the ROC curves and their 95\% confidence interval for male and female are shown in Table 6 , respectively. And MAP had a larger area under the ROC curves than SBP or DBP. Additionally, the optimal cutoff points of MAP to predict NAFLD in male and female were $88 \mathrm{mmHg}$ (with the sensitivity of 0.746 and specificity of 0.508 ) and $89 \mathrm{mmHg}$ (with the sensitivity of 0.649 and specificity of 0.619$)$, respectively.

\section{Discussion}

In this present study, we have demonstrated that MAP was one of the independent risk factors for incident NAFLD among nonobese participants with normal LDL-C levels in China. In subgroup analysis, although the incidence rate of NAFLD was significantly higher in male than female, the positive association between MAP and the incident NAFLD remained statistically significant in female, but not in male. Further, the areas under the ROC curves indicated that MAP was slightly superior to SBP or DBP for predicting NAFLD in the nonobese Chinese with normal LDL-C levels. Our results may be helpful for clinicians to identify subjects that are at a much higher risk for developing NAFLD.

Although individuals with NAFLD tend to be obese, quite a few NAFLD are nonobese population [11, 14], and unexpectedly, the proportion of nonobesity in NAFLD reached up to $75 \%$ in India [15]. The nonobese population with normal LDL-C levels in China had a $14.37 \%$ chance of developing NAFLD during five years of follow-up. Thus, it is quite necessary to determine a parameter that is reproducible, easily obtained, reliable, and practical for predicting the development of NAFLD in nonobese population with 
TABLE 3: Incidence rate of NAFLD stratified by mean arterial pressure.

\begin{tabular}{|c|c|c|c|c|}
\hline Group & Number & Number of NAFLD & Cumulative incidence (95\% CI) & Per 10,000 person-years \\
\hline Total & 16153 & 2321 & $14.37(13.68-15.06)$ & 513.17 \\
\hline Q1 & 3806 & 191 & $1.18(0.97-1.39)$ & 173.66 \\
\hline Q2 & 4414 & 473 & $2.93(2.60-3.26)$ & 388.26 \\
\hline Q3 & 3744 & 652 & $4.04(3.65-4.42)$ & 617.54 \\
\hline Q4 & 4189 & 1005 & $6.22(5.75-6.70)$ & 872.41 \\
\hline$P$ value for log-rank test & - & - & $<0.001$ & - \\
\hline Male & 8472 & 1294 & $15.27(14.57-15.99)$ & 553.40 \\
\hline Q1 & 1817 & 98 & $1.16(0.95-1.37)$ & 188.58 \\
\hline Q2 & 2247 & 244 & $2.88(2.55-3.21)$ & 397.76 \\
\hline Q3 & 2049 & 384 & $4.53(4.12-4.94)$ & 681.49 \\
\hline Q4 & 2359 & 568 & $6.70(6.21-7.19)$ & 888.49 \\
\hline$P$ value for log-rank test & - & - & $<0.001$ & - \\
\hline Female & 7681 & 1027 & $13.37(12.70-14.04)$ & 469.15 \\
\hline Q1 & 1989 & 93 & $1.21(1.00-1.43)$ & 160.68 \\
\hline Q2 & 2167 & 229 & $2.98(2.65-3.31)$ & 377.41 \\
\hline Q3 & 1695 & 268 & $3.49(3.13-3.85)$ & 543.34 \\
\hline Q4 & 1830 & 437 & $5.69(5.24-6.14)$ & 852.85 \\
\hline$P$ value for log-rank test & - & - & $<0.001$ & - \\
\hline
\end{tabular}

Abbreviations: $\mathrm{CI}=$ confidence interval; $\mathrm{NAFLD}=$ nonalcoholic fatty liver disease.

normal LDL-C. A bidirectional relationship between NAFLD and hypertension has been found by a prospective study [16]. That is to say, NAFLD can increase the risk of developing hypertension, whereas hypertension also increases the risk of developing NAFLD. Meanwhile, research achievements that elevated SBP or DBP which is independently correlated with an increased risk of the incident NAFLD in general population emerge in endlessly recent years [7-10]. Interestingly, two of the studies indicated elevated blood pressure is associated with incident NAFLD in subjects without hypertension $[8,10]$. One study by Qian et al. demonstrated that both SBP and DBP are independent risk factors for incident NAFLD in nonhypertensive subjects [10], while $\mathrm{Wu}$ et al. argued that only the elevated SBP independently increases the risk of the incidence of NAFLD in subjects with normal SBP [8]. However, as far as we know, no data is available with respect to the correlation of MAP with incident NAFLD, not to mention a comparison of MAP with SBP or DBP for the prediction of NAFLD in nonobese population. We are the first to show that the elevated MAP independently increased the risk of the incident NAFLD, with an adjusted HR (Q4 versus Q1) of 1.697 (95\% CI 1.231-2.340). After stratifying by gender, the adjusted HR increased to 2.377 (95\% CI 1.452-3.890) in women. In addition, compared with SBP or DBP, MAP did slightly better to predict NAFLD in the nonobese Chinese with normal LDL-C levels, with optimal cutoff points of $88 \mathrm{mmHg}$ in male and $89 \mathrm{mmHg}$ in female.

After dividing the subjects into 2 groups of male and female, we found that male was at more risk of incident NAFLD than female. A previous study in Korea indicated that male accounts for the majority of nonobese subjects with NAFLD, but this proportion is relatively lower than obese subjects with NAFLD [17]. To some extent, the following reasons might be used to explain the gender difference of NAFLD. As shown in Table 2, other risk factors like age, BMI, blood pressure, FPG, and TG were higher in male than in female, and conversely, the levels of HDL-C decreased in male. Moreover, endogenous estradiol could play a protective role in the development of NAFLD [18].

The specific mechanism about the relationship between MAP and the incident NAFLD remains unclear. Considering that MAP is a combination of two components of BP: SBP and DBP, the proposed pathophysiologic mechanisms like insulin resistance, sympathetic nervous system activity, and arterial stiffness which are in regard to the relationship between increased BP and NAFLD [4] may serve as possible explanations that MAP independently increased the risk of incident NAFLD. For example, insulin resistance increases BP through enhancing salt absorption and activating the sympathetic nervous system [19], and hypertension is a predictor of insulin resistance in turn [20,21], whereas insulin resistance leads to liver endothelial dysfunction and then promotes NAFLD [20, 22]. Furthermore, as a steady component, MAP is strongly associated with an increased risk of cardiovascular disease and diabetes [23, 24]. Therefore, in the nonobese population with normal LDL-C levels, MAP could be applied to identify subjects who are more susceptible to NAFLD.

There are some limitations of our study. Firstly, ultrasonography is a common way to diagnose NAFLD in epidemiological surveys but hardly assesses the severity of NAFLD. Secondly, insulin resistance plays an important role in developing NAFLD among the nonobese, but insulin levels and insulin resistance were not examined in the initial study. Thirdly, some important variables like lifestyle, smoking, and nutrition are unavailable. Lastly, the information about 


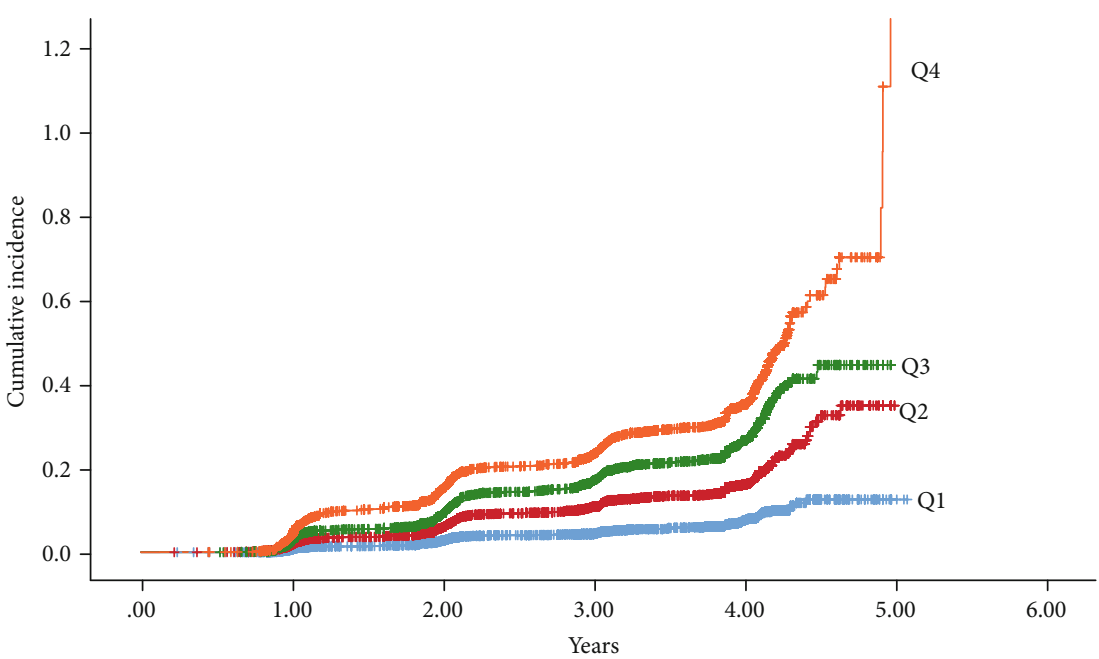

(a)

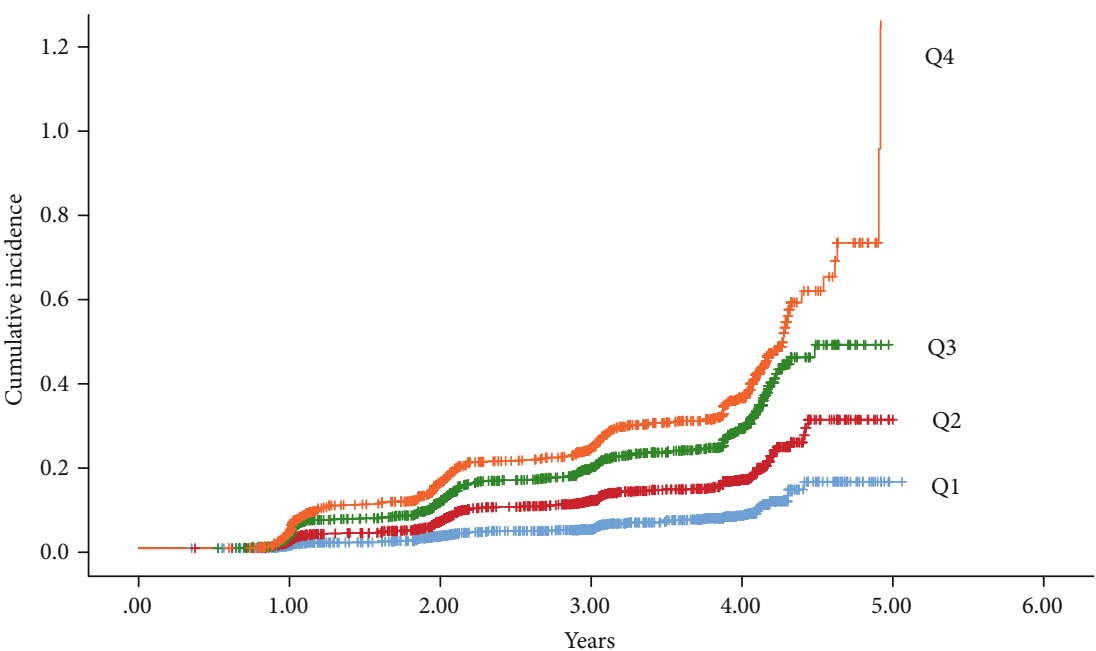

(b)

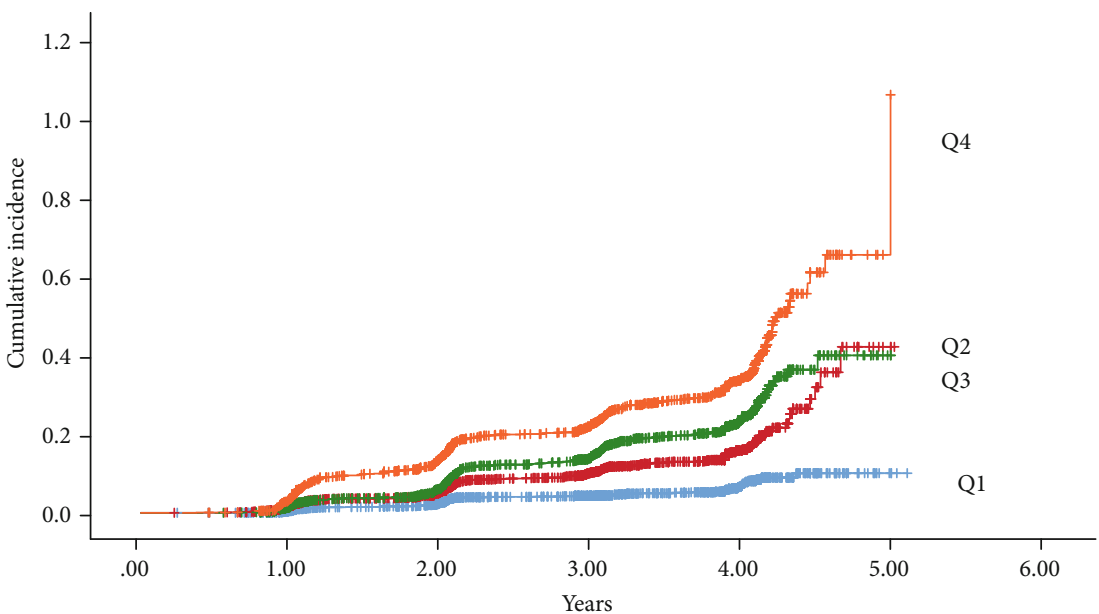

(c)

FIgURE 1: Cumulative incidence of nonalcoholic fatty liver disease (NAFLD) in the nonobese participants. (a) Cumulative incidence of NAFLD of the whole population stratified by mean arterial pressure (MAP). (b) Cumulative incidence of NAFLD of 8,472 men stratified by MAP. (c) Cumulative incidence of NAFLD of 7,681 women stratified by MAP. Q1 (58 to $<80 \mathrm{mmHg}$ ), Q2 (80 to $<88 \mathrm{mmHg}$ ), Q3 (88 to $<96 \mathrm{mmHg}$ ), and Q4 (96 to $\leq 142 \mathrm{mmHg}$ ). 
TABLE 4: Univariate Cox regression analysis for incident nonalcoholic fatty liver disease.

\begin{tabular}{|c|c|c|c|c|}
\hline \multirow{2}{*}{ Characteristics } & \multicolumn{2}{|c|}{ Univariate analysis } & \multicolumn{2}{|c|}{ Multivariate analysis } \\
\hline & HR (95\% CI) & $P$ value & $\mathrm{HR}(95 \% \mathrm{CI})$ & $P$ value \\
\hline Q1 & Ref & & Ref & \\
\hline Q2 & $2.273(1.921-2.689)$ & $<0.001$ & $1.328(1.072-1.647)$ & 0.010 \\
\hline Q3 & $3.613(3.075-4.245)$ & $<0.001$ & $1.625(1.276-2.069)$ & $<0.001$ \\
\hline Q4 & $5.083(4.354-5.934)$ & $<0.001$ & $1.697(1.231-2.340)$ & 0.001 \\
\hline$P$ for trend & & $<0.001$ & & 0.001 \\
\hline Age & $1.006(1.004-1.009)$ & $<0.001$ & $1.008(1.004-1.011)$ & 0.000 \\
\hline Gender & $1.181(1.088-1.282)$ & $<0.001$ & $0.933(0.850-1.025)$ & 0.147 \\
\hline BMI & $1.815(1.765-1.866)$ & $<0.001$ & $1.535(1.485-1.587)$ & $<0.001$ \\
\hline SBP & $1.023(1.021-1.025)$ & $<0.001$ & $0.993(0.988-0.997)$ & 0.002 \\
\hline DBP & $1.046(1.042-1.050)$ & $<0.001$ & $1.007(0.999-1.016)$ & 0.089 \\
\hline ALP & $1.008(1.007-1.009)$ & $<0.001$ & $1.005(1.003-1.007)$ & $<0.001$ \\
\hline ALT & $1.007(1.006-1.008)$ & $<0.001$ & $1.014(1.011-1.017)$ & $<0.001$ \\
\hline AST & $1.009(1.007-1.011)$ & $<0.001$ & $0.977(0.968-0.985)$ & $<0.001$ \\
\hline ALB & $1.005(0.988-1.021)$ & 0.586 & & \\
\hline GLB & $1.008(0.997-1.020)$ & 0.166 & $0.999(0.987-1.011)$ & 0.860 \\
\hline TB & $0.998(0.989-1.008)$ & 0.695 & & \\
\hline BUN & $0.933(0.903-0.963)$ & $<0.001$ & $0.828(0.796-0.862)$ & $<0.001$ \\
\hline $\mathrm{Cr}$ & $1.005(1.004-1.005)$ & $<0.001$ & $1.002(1.001-1.004)$ & $<0.001$ \\
\hline UA & $1.005(1.005-1.006)$ & $<0.001$ & $0.999(0.999-1.000)$ & 0.035 \\
\hline FPG & $1.297(1.268-1.327)$ & $<0.001$ & $1.210(1.170-1.251)$ & $<0.001$ \\
\hline $\mathrm{TC}$ & $1.300(1.238-1.365)$ & $<0.001$ & $0.776(0.690-0.872)$ & $<0.001$ \\
\hline TG & $1.204(1.192-1.216)$ & $<0.001$ & $1.234(1.178-1.293)$ & $<0.001$ \\
\hline HDL-C & $0.278(0.245-0.316)$ & $<0.001$ & $0.797(0.664-0.956)$ & 0.014 \\
\hline LDL-C & $1.950(1.774-2.143)$ & $<0.001$ & $1.850(1.551-2.207)$ & $<0.001$ \\
\hline
\end{tabular}

Note: ALB and TB were not included into multivariate analysis for their $P$ value more than 0.2 in the univariate cox analysis. Abbreviations: $\mathrm{BMI}=$ body mass index; $\mathrm{SBP}=$ systolic blood pressure; $\mathrm{DBP}=$ diastolic blood pressure; $\mathrm{ALP}=$ alkaline phosphatase; $\mathrm{ALT}=$ alanine aminotransferase; $\mathrm{AST}=\mathrm{aspartate}$ aminotransferase; $\mathrm{ALB}=$ albumin; $\mathrm{GLB}=$ globulin; $\mathrm{TB}=$ total bilirubin; $\mathrm{BUN}=$ blood urea nitrogen; $\mathrm{Cr}=\mathrm{creatinine} ; \mathrm{UA}=\mathrm{uric}$ acid; $\mathrm{FPG}=$ fasting plasma glucose; TC = total cholesterol; TG = triglyceride; HDL-C = high density lipoprotein cholesterol; LDL-C = low-density lipoprotein cholesterol; HR = hazard ratio; $\mathrm{CI}=$ confidence interval.

TABLE 5: Univariate and multivariate Cox regression analysis for incident nonalcoholic fatty liver disease, stratified by gender.

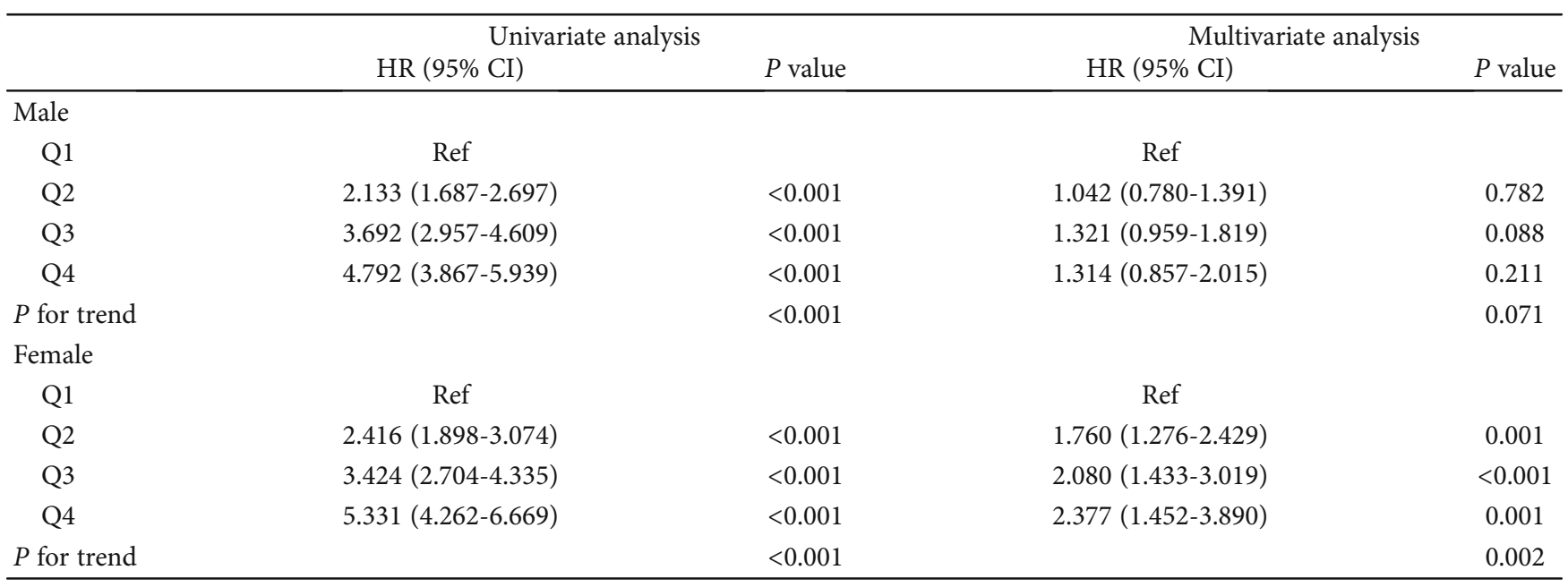

Abbreviations: $\mathrm{HR}=$ hazard ratio; $\mathrm{CI}=$ confidence interval. 


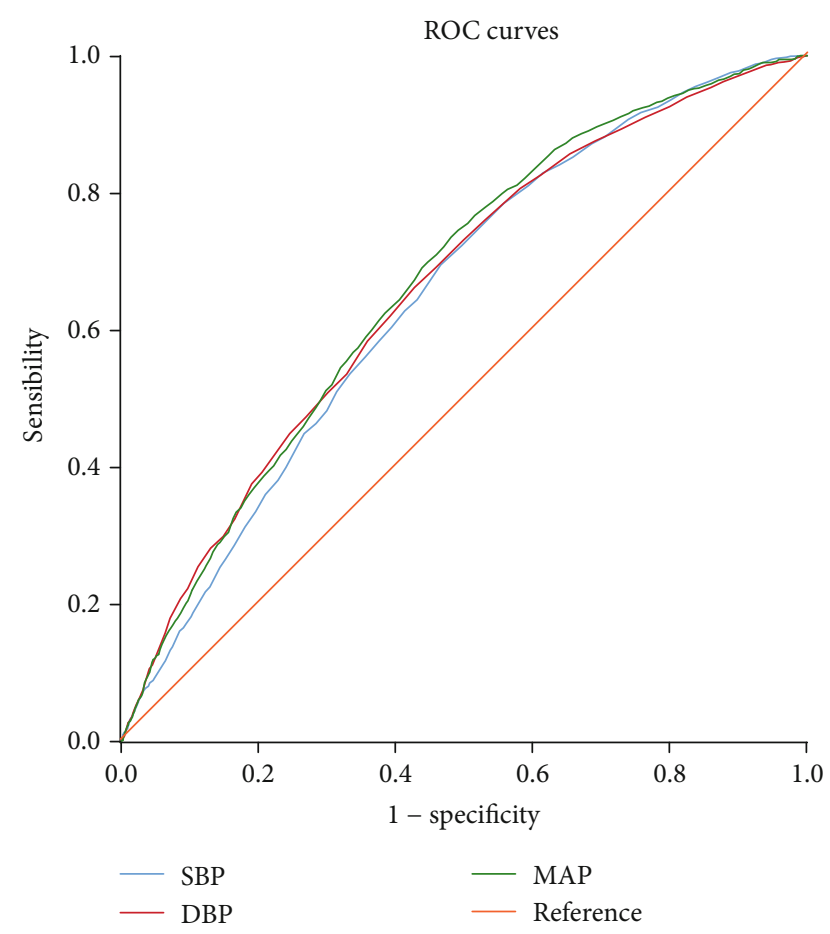

FIGURE 2: ROC curves for systolic blood pressure (SBP), diastolic blood pressure (DBP), and mean arterial pressure (MAP) for predicting nonalcoholic fatty liver disease in the 8,472 men.

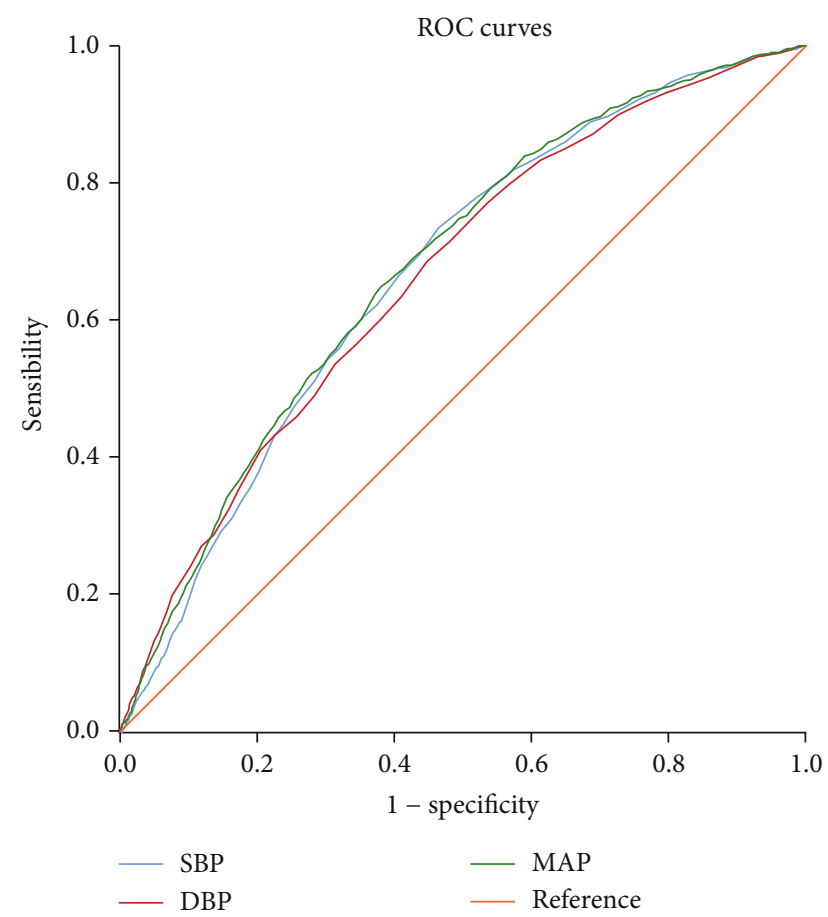

FIGURE 3: ROC curves for systolic blood pressure (SBP), diastolic blood pressure (DBP), and mean arterial pressure (MAP) for predicting nonalcoholic fatty liver disease in the 7,681 women.
TABLE 6: The area under the ROC curves and their 95\% confidence interval for male and female.

\begin{tabular}{lcc}
\hline & \multicolumn{2}{c}{ Area under the curve } \\
& (95\% confidence interval) \\
& Male & Female \\
\hline Systolic blood pressure & $0.648(0.633-0.663)$ & $0.668(0.652-0.685)$ \\
Diastolic blood pressure & $0.659(0.644-0.675)$ & $0.663(0.646-0.680)$ \\
Mean arterial pressure & $0.666(0.651-0.681)$ & $0.676(0.660-0.693)$ \\
\hline
\end{tabular}

changes in anthropometric parameters over time and the status of appearance of other components of metabolic syndrome during the study period were also unavailable.

\section{Conclusion}

Increased MAP is independently associated with NAFLD in nonobese female with normal LDL-C levels, but not in nonobese male, which behaves slightly better in NAFLD prediction. MAP may be clinically useful in identifying people at a higher risk of developing NAFLD.

\section{Data Availability}

The data that support the findings of this study are openly available in Dryad repository at 10.5061/dryad.1n6c4.

\section{Ethical Approval}

This study did a secondary analysis data obtained in a retrospective study by Sun et al. They clearly stated that their study was approved by the Ethics Committee of Wenzhou People's Hospital. All data were anonymously linked and no personal identifiers were available to researchers.

\section{Consent}

Data were drawn from anonymous regional database, and informed consent was not required.

\section{Conflicts of Interest}

The authors declare that there is no conflict of interest regarding the publication of this article.

\section{Acknowledgments}

The authors are very grateful to the data providers of the study.

\section{References}

[1] Z. M. Younossi, A. B. Koenig, D. Abdelatif, Y. Fazel, L. Henry, and M. Wymer, "Global epidemiology of nonalcoholic fatty liver disease-meta-analytic assessment of prevalence, incidence, and outcomes," Hepatology, vol. 64, no. 1, pp. 73-84, 2016.

[2] A. R. Araújo, N. Rosso, G. Bedogni, C. Tiribelli, and S. Bellentani, "Global epidemiology of non-alcoholic fatty liver 
disease/non-alcoholic steatohepatitis: what we need in the future," Liver International, vol. 38, Suppl 1, pp. 47-51, 2018.

[3] D. N. Amarapurkar, E. Hashimoto, L. A. Lesmana et al., "How common is non-alcoholic fatty liver disease in the Asia?Pacific region and are there local differences?," Journal of Gastroenterology and Hepatology, vol. 22, no. 6, pp. 788-793, 2007.

[4] D. Oikonomou, G. Georgiopoulos, V. Katsi et al., "Non-alcoholic fatty liver disease and hypertension: coprevalent or correlated?," European Journal of Gastroenterology \& Hepatology, vol. 30, no. 9, pp. 979-985, 2018.

[5] K. C. Sung and S. H. Kim, "Interrelationship between fatty liver and insulin resistance in the development of type 2 diabetes," The Journal of Clinical Endocrinology and Metabolism, vol. 96, no. 4, pp. 1093-1097, 2011.

[6] G. Targher, C. D. Byrne, A. Lonardo, G. Zoppini, and C. Barbui, "Non-alcoholic fatty liver disease and risk of incident cardiovascular disease: a meta-analysis," Journal of Hepatology, vol. 65, no. 3, pp. 589-600, 2016.

[7] R. L. Vasunta, Y. A. Kesäniemi, A. S. Ylitalo, and O. H. Ukkola, "High ambulatory blood pressure values associated with non-alcoholic fatty liver in middle-aged adults," Journal of Hypertension, vol. 30, no. 10, pp. 2015-2019, 2012.

[8] S.-J. Wu, H. Zou, G.-Q. Zhu et al., "Increased levels of systolic blood pressure within the normal range are associated with significantly elevated risks of nonalcoholic fatty liver disease," Medicine, vol. 94, no. 19, p. e842, 2015.

[9] H. Ge, J. Chen, Y. M. Sun, and W. H. Ling, "Prevalence of nonalcoholic fatty liver disease and its correlated risk factors in health population in Guangzhou," Zhonghua Yi Xue Za Zhi, vol. 96, no. 46, pp. 3706-3709, 2016.

[10] L. Y. Qian, J. F. Tu, Y. H. Ding et al., “Association of blood pressure level with nonalcoholic fatty liver disease in nonhypertensive population: normal is not the new normal," Medicine, vol. 95, no. 29, article e4293, 2016.

[11] D. Q. Sun, S. J. Wu, W. Y. Liu et al., "Association of lowdensity lipoprotein cholesterol within the normal range and NAFLD in the non-obese Chinese population: a crosssectional and longitudinal study," BMJ Open, vol. 6, no. 12, article e013781, 2016.

[12] D. Q. Sun, S. J. Wu, W. Y. Liu et al., Data from: Association of Low-density Lipoprotein Cholesterol within the Normal Range and NAFLD in the Non-obese Chinese Population: A CrossSectional and Longitudinal Study, Dryad, 2016.

[13] M. D. Zeng, Y. M. Li, C. W. Chen et al., "Guidelines for the diagnosis and treatment of alcoholic liver disease," Journal of Digestive Diseases, vol. 9, no. 2, pp. 113-116, 2008.

[14] C. J. Liu, "Prevalence and risk factors for non-alcoholic fatty liver disease in Asian people who are not obese," Journal of Gastroenterology and Hepatology, vol. 27, no. 10, pp. 15551560, 2012.

[15] K. Das, K. Das, P. S. Mukherjee et al., "Nonobese population in a developing country has a high prevalence of nonalcoholic fatty liver and significant liver disease," Hepatology, vol. 51, no. 5, pp. 1593-1602, 2010.

[16] J. Ma, S. J. Hwang, A. Pedley et al., "Bi-directional analysis between fatty liver and cardiovascular disease risk factors," Journal of Hepatology, vol. 66, no. 2, pp. 390-397, 2017.

[17] H. C. Cho, "Prevalence and factors associated with nonalcoholic fatty liver disease in a nonobese Korean population," Gut and Liver, vol. 10, no. 1, pp. 117-125, 2016.
[18] V. Jaruvongvanich, A. Sanguankeo, T. Riangwiwat, and S. Upala, "Testosterone, sex hormone-binding globulin and nonalcoholic fatty liver disease: a systematic review and meta-analysis," Annals of Hepatology, vol. 16, no. 3, pp. 382394, 2017.

[19] G. M. Reaven and B. B. Hoffman, "A role for insulin in the aetiology and course of hypertension?" Lancet, vol. 2, no. 8556, pp. 435-437, 1987.

[20] G. Donati, B. Stagni, F. Piscaglia et al., "Increased prevalence of fatty liver in arterial hypertensive patients with normal liver enzymes: role of insulin resistance," Gut, vol. 53, no. 7, pp. 1020-1023, 2004.

[21] E. Ferrannini, G. Buzzigoli, R. Bonadonna et al., "Insulin resistance in essential hypertension," The New England Journal of Medicine, vol. 317, no. 6, pp. 350-357, 1987.

[22] E. Bugianesi, A. Gastaldelli, E. Vanni et al., "Insulin resistance in non-diabetic patients with non-alcoholic fatty liver disease: sites and mechanisms," Diabetologia, vol. 48, no. 4, pp. 634642, 2005.

[23] H. D. Sesso, M. J. Stampfer, B. Rosner et al., "Systolic and diastolic blood pressure, pulse pressure, and mean arterial pressure as predictors of cardiovascular disease risk in men," Hypertension, vol. 36, no. 5, pp. 801-807, 2000.

[24] M. Janghorbani and M. Amini, "Comparison of systolic and diastolic blood pressure with pulse pressure and mean arterial pressure for prediction of type 2 diabetes: the Isfahan diabetes prevention study," Endokrynologia Polska, vol. 62, no. 4, pp. 324-330, 2011. 


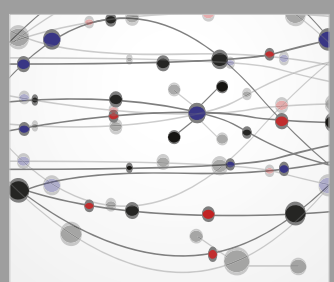

The Scientific World Journal
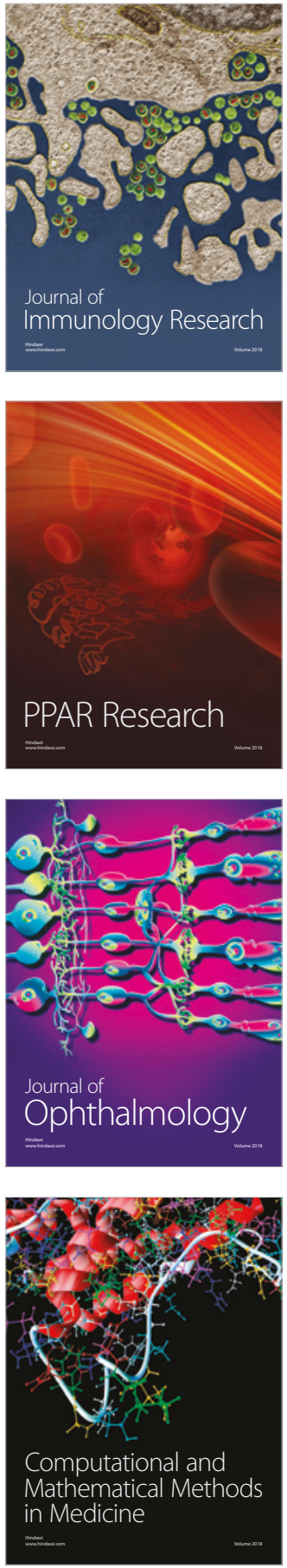

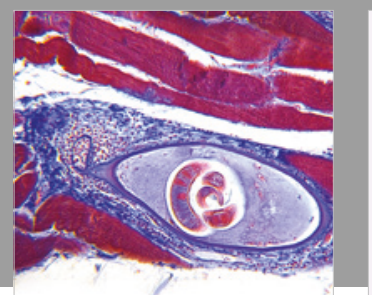

Gastroenterology Research and Practice

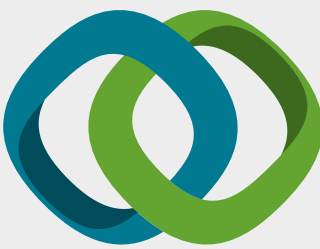

\section{Hindawi}

Submit your manuscripts at

www.hindawi.com
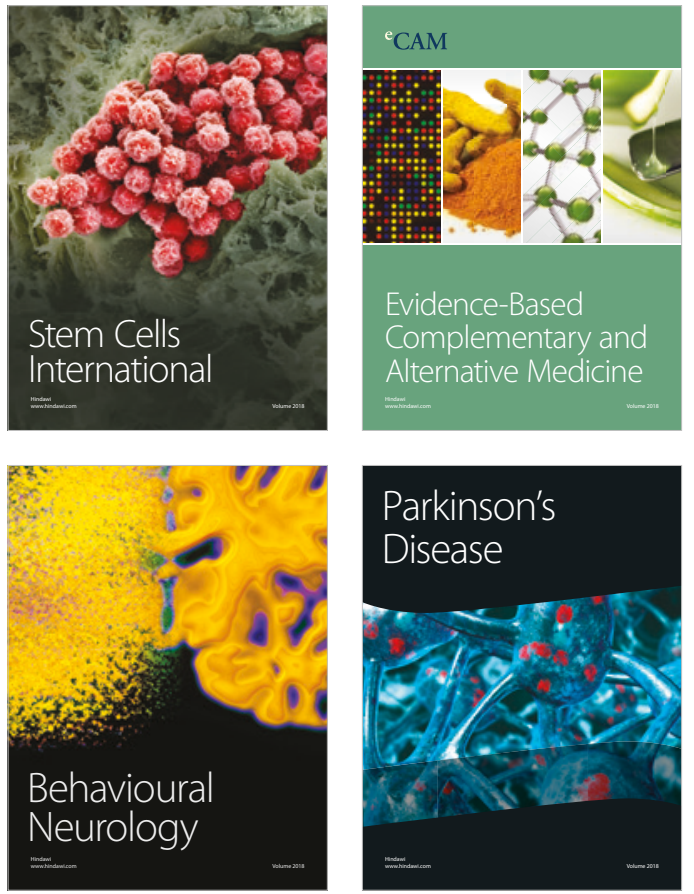

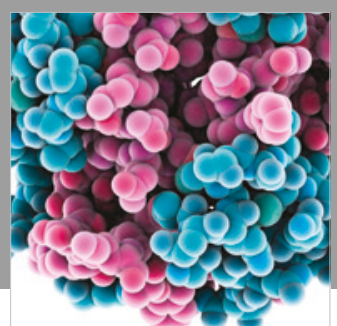

ournal of

Diabetes Research

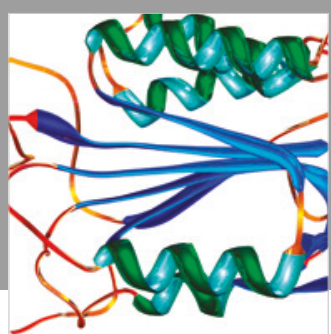

Disease Markers
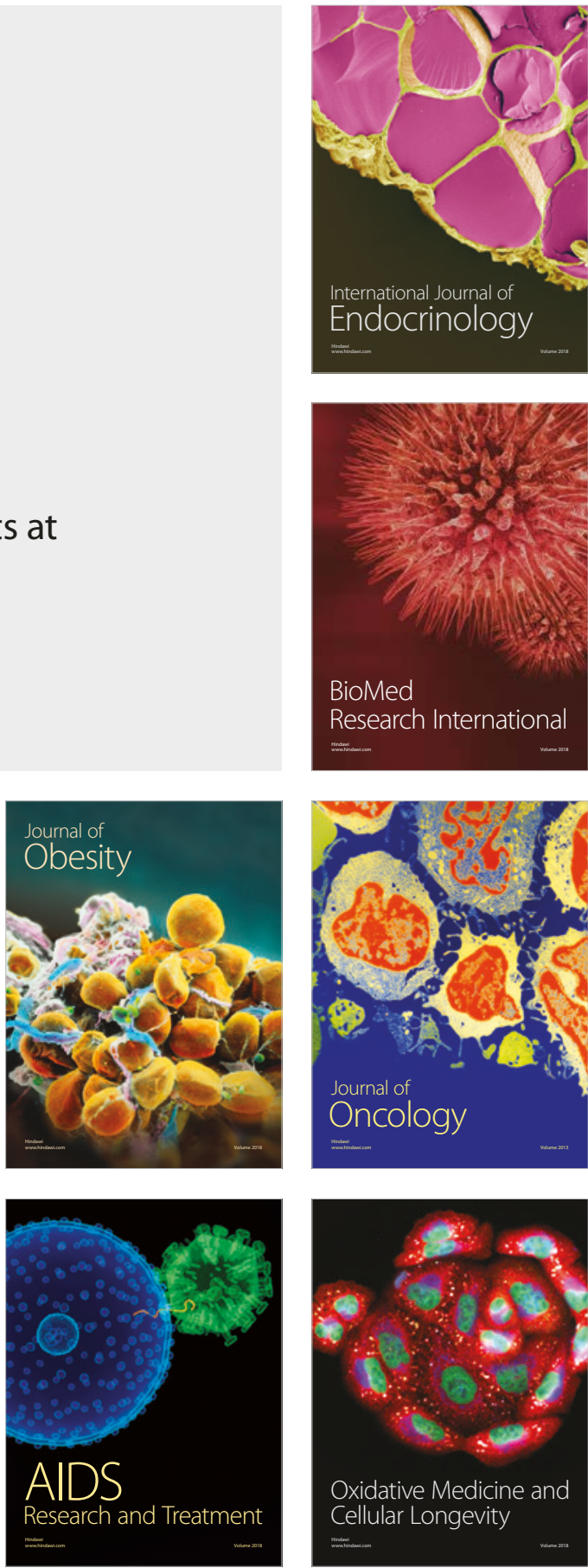